\title{
Abdominoplastias: neo-onfaloplastia sem cicatriz e sem excisão de gordura
}

\author{
Abdominoplasty: neoomphaloplasty without scar or fat excision
}

Joseph Alexander

Abreu $\mathrm{NG}^{1}$

Trabalho realizado no Perfecta Day Hospital, Diadema, SP,

Brasil.

Artigo submetido pelo SGP (Sistema de Gestão de Publicações) da RBCP.

Artigo recebido: 7/4/2010 Artigo aceito: $11 / 8 / 2010$

\begin{abstract}
RESUMO
Introdução: A cicatriz do umbigo é a única do corpo aceita culturalmente, cuja dismorfia ou ausência chama a atenção negativamente. As distorções dessa cicatriz, que ainda ocorrem com frequência nas abdominoplastias, determinaram a elaboração de inúmeros procedimentos técnicos com a finalidade estética de imitar o seu aspecto natural. Apresentamos mais uma opção sobre neo-onfaloplastia no decurso das plásticas de abdome, mediante uma técnica de fácil execução, com efeito estético próximo ao umbigo natural e sem cicatrizes cutâneas. Método: No decurso da abdominoplastia, o umbigo e o seu pedículo são amputados e a derme da pele na área de projeção do neoumbigo é suturada diretamente no plano da fáscia muscular abaixo, após divulsão tecido adiposo local para produzir a necessária umbilicação sem cicatriz. Resultados: No período de nove anos, foram operados 316 pacientes com a utilização desta técnica, com constante aspecto natural e mínimos efeitos pouco estéticos.
\end{abstract}

Descritores: Abdome/cirurgia. Umbigo/cirurgia. Cirurgia plástica.

\section{SUMMARY}

Introduction: Under the cultural aspect the umbilicus is the only scar accepted in the body, its dysmorphia or absence is noted negatively. Distortions of this scar are still frequent in the abdominoplasties determined by several different aesthetic technical procedures to imitate its natural aspect. The scope of this presentation is another attempt to obtain a neoomphaloplasty based on an easy technical procedure, with a quite natural final aspect and without scars. Methods: During the abdominoplasty, the umbilicus and its stalk are amputated and the dermis of the skin over the future projection is sutured directly to the fascia below, after the local fat excesses be divulsioned to produce the natural navel aspect without scar. Results: Along the last nine years, 319 patients were operated by this technique, with a natural aspect and minimal unaesthetic effects.

Descriptors: Abdomen/surgery. Umbilicus/surgery. Surgery, plastic.

1. Cirurgião Plástico; Membro titular da Sociedade Brasileira de Cirurgia Plástica. 


\section{INTRODUÇÃO}

A cicatriz umbilical está posicionada na linha média da parede abdominal anterior, cerca de 18 a $23 \mathrm{~cm}$ da comissura vulvar, com variação de $4 \mathrm{~cm}$ acima a $2 \mathrm{~cm}$ abaixo da linha horizontal que passa pelas duas espinhas ilíacas ânterosuperiores. Toma a forma circular ou discretamente alongada verticalmente, com diâmetro médio de $1 \mathrm{~cm}$ e profundidade variável, de acordo com a espessura do panículo adiposo. Sofre modificações nas suas características básicas, de acordo com as alterações da parede na região peri-umbilical e com a flacidez da pele. Não apresenta nenhuma função após o nascimento.

As abdominoplastias em uso nas últimas décadas estão baseadas no reimplante do pedículo do umbigo na sua primitiva posição, após a ressecção dos excessos cutâneos. Esta manobra tem determinado uma cicatriz que circunda a ilha de pele deixada na sua extremidade na nova sutura, ao ser exteriorizado. Considerando-se a exposição da parede abdominal anterior e a maior exposição do umbigo com o uso de trajes de banho exíguos, várias técnicas de umbilicoplastia passaram a ser elaboradas para minimizar a presença de cicatriz visível. Vários autores ${ }^{1-7}$ registraram as primeiras tentativas nas décadas de 1960 a 1980, com cicatriz externamente pouco visível. Onfaloneoplastias com retalhos locais de formas diversas, associados ou não com enxertos de pele, foram também realizadas. Kirianoff ${ }^{8}$ e Schoeller et al. ${ }^{9}$ realizam a umbilicoplastia sem cicatriz, mediante a desepitelização da ilha de pele umbilical, fixando o coto na aponeurose. Após esta manobra, o tecido adiposo no local da posição do novo umbigo é ressecado e quatro pontos cardinais são aplicados, fixando a derme do retalho abdominal ao coto desepitelizado. Santanelli et al. ${ }^{10}$, partindo da premissa que o umbigo é uma cicatriz gerada por segunda intenção, amputam o seu pedículo, incisam a pele $1 \mathrm{~cm}$ transversalmente, na linha de posicionamento do futuro neoumbigo, suturando as bordas diretamente na linha alba, deixando cicatrizar por segunda intenção. Marconi ${ }^{11}$, Onishi et al. ${ }^{12} \mathrm{e}$ Franco \& Franco $^{13}$ seguem a linha de onfaloneoplastia com mínimo de cicatriz. Em 2008, Amud ${ }^{14}$ publica um método de neo-onfaloplastia sem cicatriz. A partir de 2001, elaboramos um procedimento para a neo-onfaloplastia, na tentativa de obter um resultado com aspecto semelhante ao do umbigo natural, de fácil execução e sem cicatrizes, com mínimo índice de complicações.

\section{MÉTODO}

No período de abril de 2001 a maio de 2009 , foram realizadas 316 abdominoplastias com a utilização desta técnica de neo-onfaloplastia, em pacientes do sexo feminino, com idade entre 32 e 53 anos (Tabela 1).
Tabela 1 - Relação entre idade e o número total de pacientes e porcentagem.

\begin{tabular}{l|c|c}
\hline Faixa Etária & $\mathbf{N}^{\mathbf{0}}$ Pacientes & Porcentagem \\
\hline $32-37$ anos & 51 & 16,6 \\
\hline $38-42$ anos & 84 & 27,7 \\
\hline $43-47$ anos & 112 & 36,1 \\
\hline $48-52$ anos & 69 & 22,2 \\
\hline
\end{tabular}

Os abdomes operados pertenceram ao grupo IV da classificação de Bozola \& Psillakis ${ }^{15}, 11 \%$ das pacientes apresentavam umbigo com desvio de 5 a $10 \mathrm{~mm}$ da linha média e $15 \%$, hérnia umbilical.

\section{Técnica Operatória}

Paciente sob condições operatórias, a abdominoplastia foi realizada mediante incisão pubiana transversal baixa, seguida das etapas de dissecção cutânea até o xifóide e plicatura dos músculos retos. O pedículo do umbigo foi amputado parcialmente e o coto suturado no nível da fáscia dos músculos retos, mediante pontos em $\mathrm{U}$, com o nó voltado para a parte subfacial (Figura 1). Hérnias, quando presentes, foram reduzidas neste tempo operatório. Em seguida, os excessos cutâneos foram ressecados e um ponto de reparo provisório fixou a linha média do retalho cutâneo abdominal ao púbis. Uma pinça longa com tinta na extremidade demarcou na pele a futura posição do neoumbigo (Figura 2). O retalho cutâneo liberado do seu ponto de reparo, mediante manobra digital, foi evertido e a pele pressionada no ponto de transmissão da posição do neoumbigo. Ainda sob compressão digital externa, foi realizada demarcação em cruz com tinta no celular subcutâneo, seguida da sua divulsão até atingir o derma, sem a sua ressecção, para a seguir serem dados os pontos que fixaram o derma na fáscia imediatamente abaixo (Figura 3). Foram aplicados 6 pontos isolados com fio mononylon 3-0, que uniram o derma na área de fixação do neoumbigo na fáscia imediatamente abaixo, segundo um hexágono com dois tipos de forma: Tipo 1 - pontos equidistantes (Figura 4) e Tipo 2 com dois pontos na linha vertical mais distantes (Figura 5). A seguir, os demais tempos operatórios seguiram a rotina da abdominoplastia até o seu término.

\section{RESULTADOS}

A avaliação do umbigo submetido a este tipo de procedimento tem confirmado resultados com elevado nível de qualidade estética, pelas suas características naturais, pela ausência de cicatrizes, pela fácil execução e pelo baixo índice de complicações. 

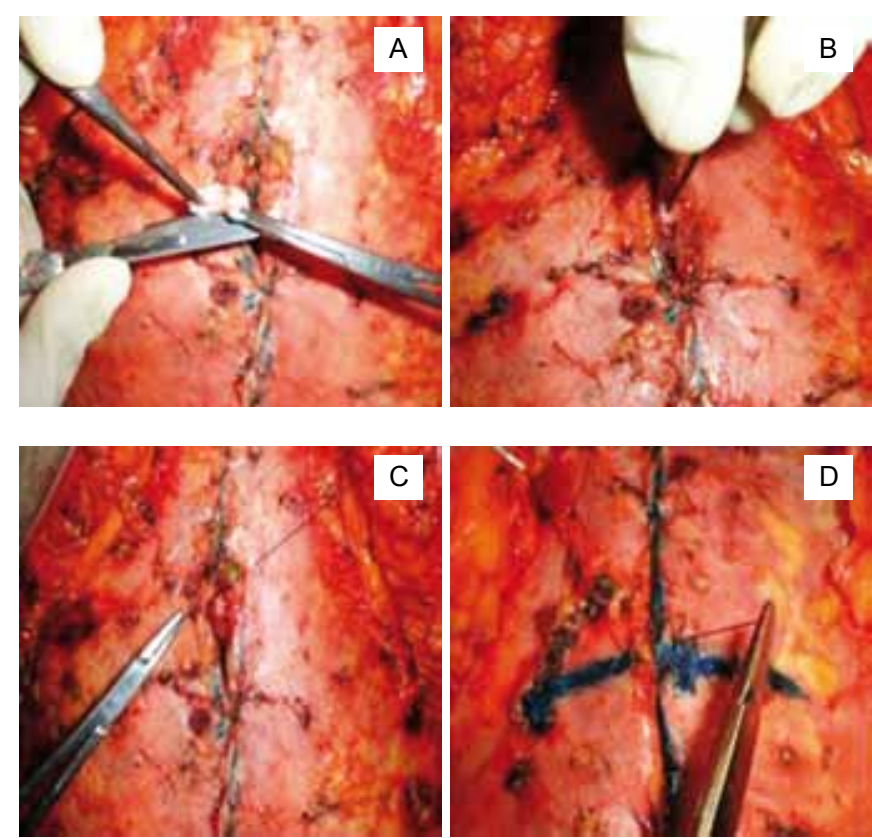

Figura 1 - A: Amputação da ilha de pele do pedículo umbilical após plicatura da fáscia dos músculos retos. B, C e D: Fixação do pediculo umbilical com fio inabsorvível na linha média com pontos em $U$.

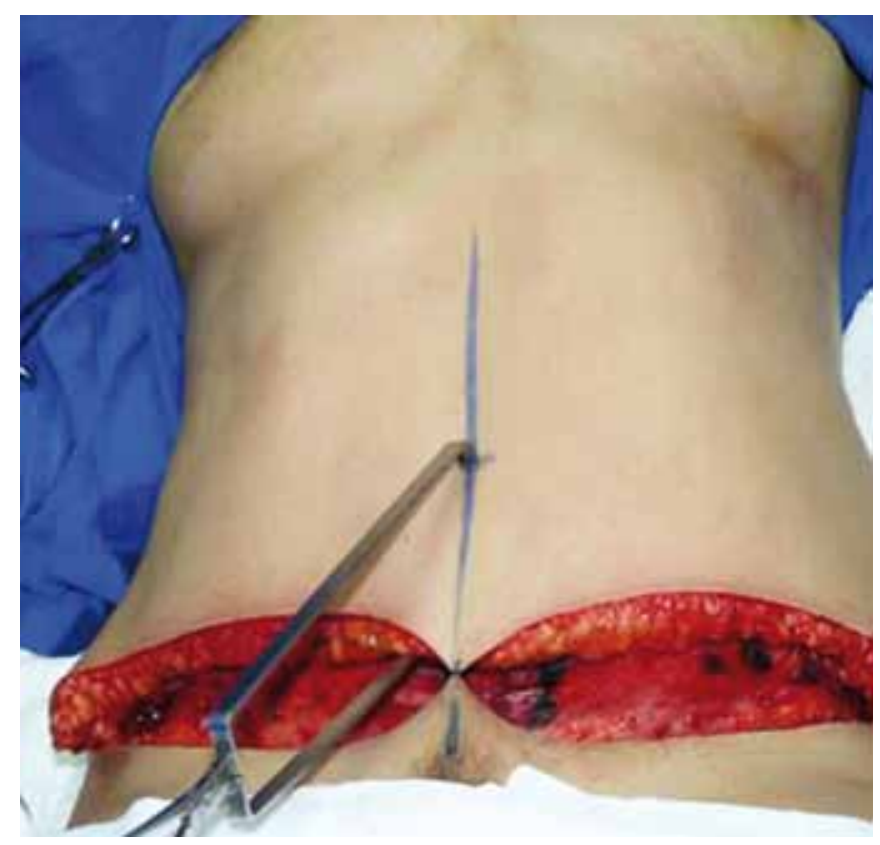

Figura 2 - Demarcação cutânea na posição correta do neoumbigo e do tecido adiposo imediatamente abaixo utilizando tinta azul nas extremidades de uma pinça longa.
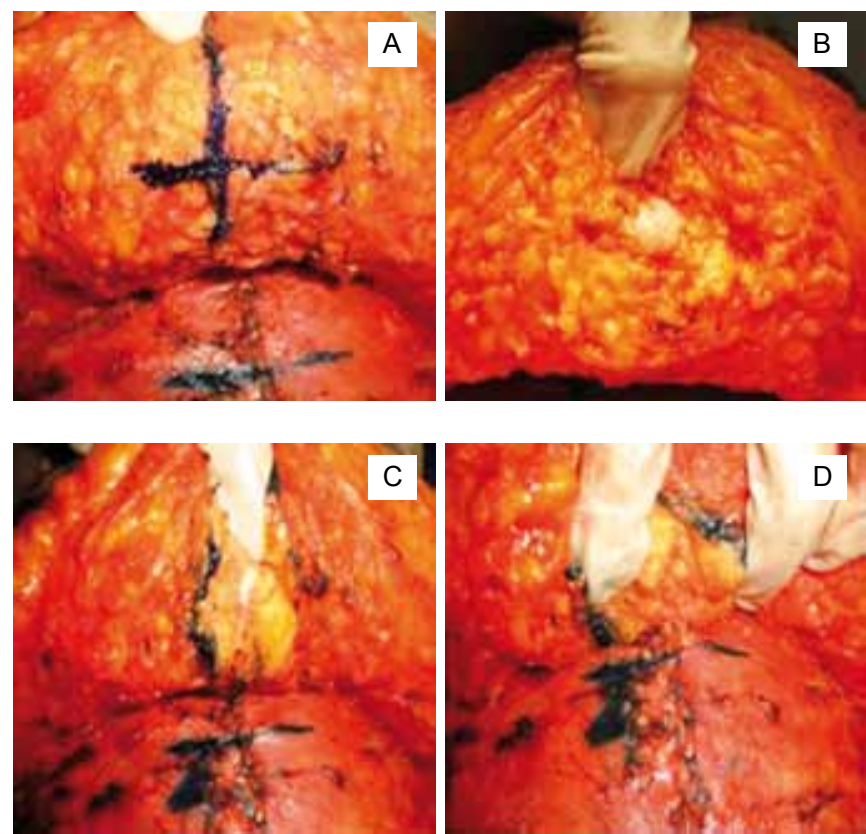

Figura 3 - A, B, C e D: Detalhes da demarcação em cruz no celular subcutâneo, seguida da divulsão sem ressecção do tecido adiposo até a exposição do derma, e pontos para ancorar o derma à fáscia muscular, mediante pontos isolados com mononylon 3-0.
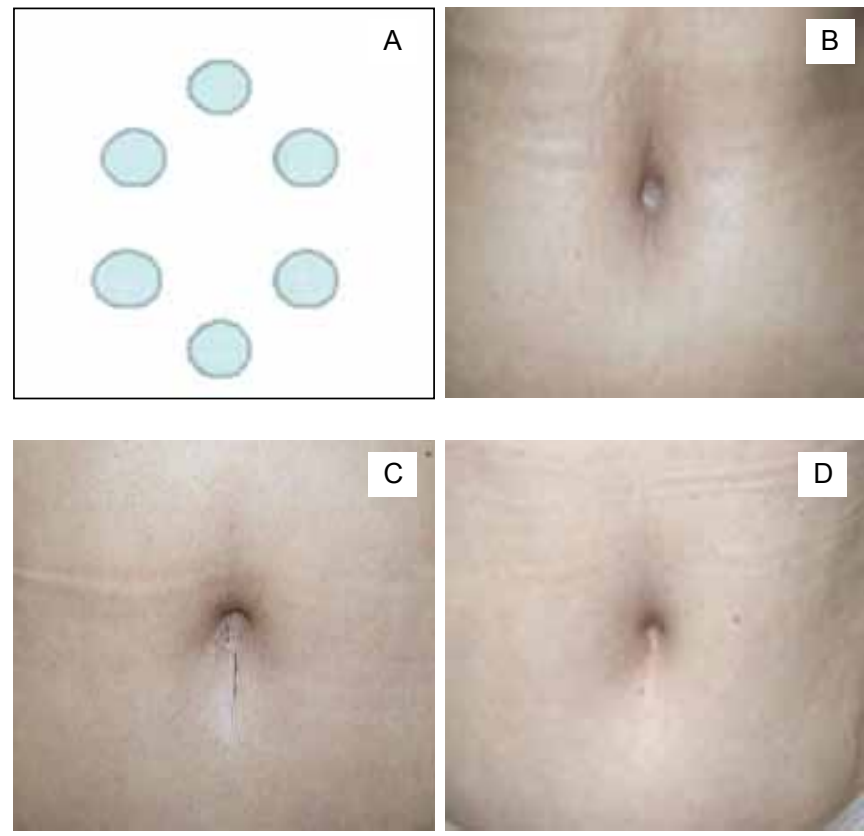

Figura 4 - A, B, C e D: Aplicação de 6 pontos de fixação segundo a forma de hexágono. Quando equidistantes (tipo 1), a fixação determina a forma circular do umbigo. 

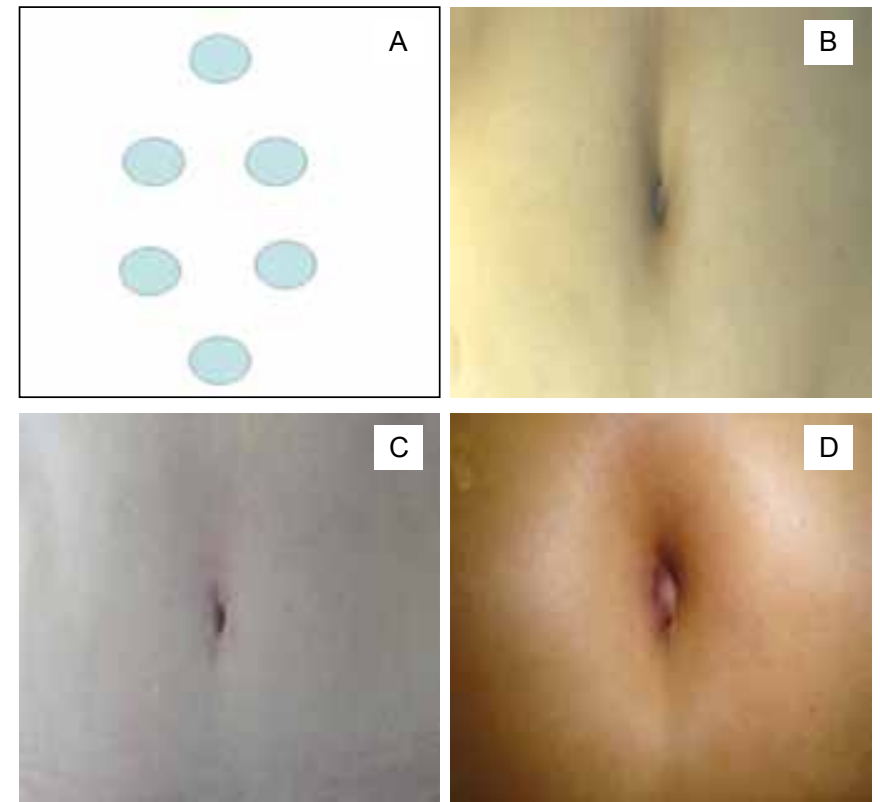

Figura 5 - A, B, C e D: Quando os pontos no eixo longitudinal estão mais afastados alguns milímetros (tipo 2) em comparação ao tipo 1, o umbigo torna-se mais alongado.
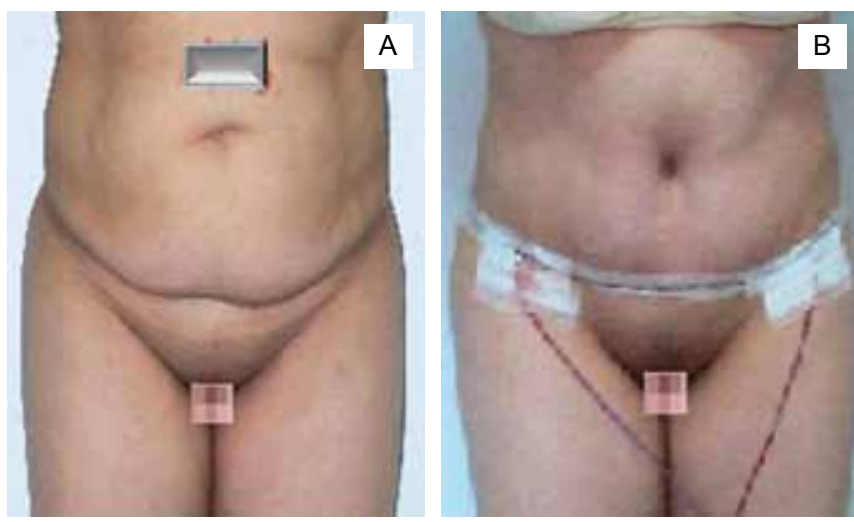

Figura 6 - A e B: Paciente de 40 anos de idade, (A) vista anterior do pré e do pós-operatório (B) de 24 horas de abdominoplastia

tipo pubiana transversal baixa, com neoumbigo (tipo 1) de formato circular, sem curativo e sem os problemas inerentes às suturas cutâneas.
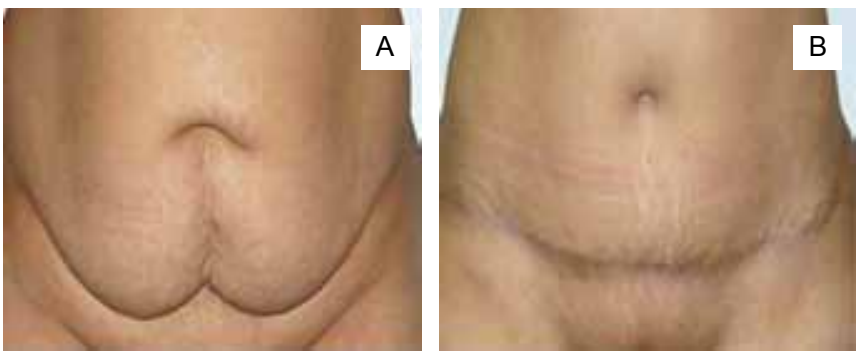

Figura 7 - A e B: Paciente de 44 anos de idade, aspecto pré e pósoperatório de 13 meses de neoumbigo tipo 1, com formato circular.
Tabela 2 - Complicações.

\begin{tabular}{l|c|c}
\hline Tipo & $\mathbf{N}^{\mathbf{0}}$ Pacientes & Porcentagem \\
\hline Deiscência da sutura & 1 & 0,31 \\
\hline Epiteliose & 31 & 9,53 \\
\hline Aplanamento & 5 & 1,58 \\
\hline Hiperpigmentação & 5 & 1,58 \\
\hline Hipertrofia cicatricial & 1 & 0,31 \\
\hline
\end{tabular}

Dentre as 316 pacientes operadas, 37 apresentaram evolução desfavorável, porém somente 7 necessitaram de reintervenção cirúrgica (Tabela 2). Em um dos casos, a paciente apresentou crises de tosse no pós-operatório imediato, desfazendo a fixação do umbigo, além de dois pontos da plicatura da fáscia dos músculos retos. Foi feita a reintervenção, que consistiu na abertura da incisão abdominal e reaplicação dos pontos de plicatura e refixação do neoumbigo. Este procedimento foi realizado um dia após a cirurgia, teve a duração de 40 minutos, e foi executado sob sedação e anestesia local. Não houve consequências. Em cinco outros casos, houve aplanamento do umbigo; quatro deles tratados com sutura em bolsa no rodete umbilical, e no outro, fizemos nova neo-onfaloplastia. A epiteliose peri-umbilical esteve presente em 31 dos casos, manifestando-se por feridas lineares de 1 a $2,5 \mathrm{~cm}$. Estas pacientes foram tratadas apenas com curativo, sendo que, em um caso, a epiteliose evoluiu para hipertrofia cicatricial, e cinco, para hiperpigmentação. Atribuímos a presença de epitelióse ao aumento de tensão dos pontos de fixação do retalho na fáscia abdominal e à exposição muito grande da derme. Como prevenção a essa complicação, devemos realizar a incisão em cruz no subcutâneo, o suficiente para distribuir bem a tensão, para que esta não fique concentrada apenas na região umbilical, além de não realizar uma divulsão muito ampla do subcutâneo.

\section{DISCUSSÃO}

É significativa a constante qualidade dos resultados desta técnica comparada às demais observadas, em que uma ilha de pele na extremidade do umbigo é suturada na pele. A diversificação dos efeitos pouco estéticos, como presença de cicatriz nitidamente visível, dimensões variadas da ilha de pele e falta de umbilicação, mostram as dificuldades de manipulação no uso dessas técnicas, que vão desde as estenoses até amplas "moedas" cicatriciais na superfície da pele.

Outro detalhe na técnica proposta é o conforto e a não necessidade de retirada de pontos, tampouco eventuais riscos de deiscências de sutura, inflamações, secreções ou mesmo 

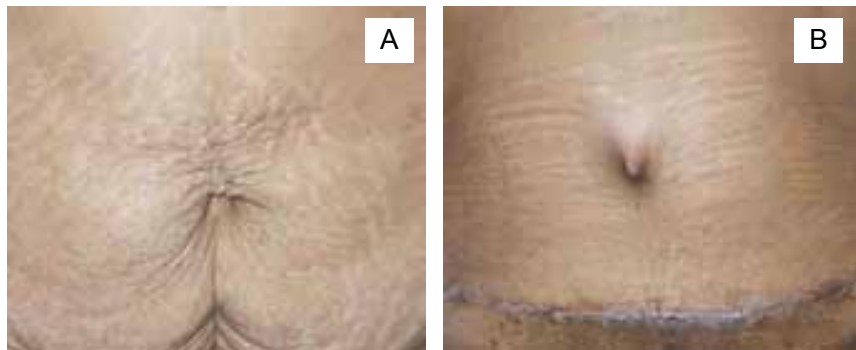

Figura 8 - A e B: Paciente de 39 anos de idade, foto aproximada do pré e pós-operatório de 7 dias tipo 2, com aspecto íntegro da pele e formato discretamente alongado no eixo vertical do umbigo.

infecções, com ou sem necrose do pedículo, que podem ou não determinar alterações secundárias na qualidade estética do umbigo. $\mathrm{Na}$ análise técnica, a ressecção da ilha de pele na extremidade livre do umbigo é necessária para evitar o aparecimento de cistos e granulomas. A seguir, a sutura do seu pedículo juntamente com a aponeurose dos músculos retos na linha alba constitui uma manobra de rotina.

A divulsão do tecido celular subcutâneo, sem a sua ressecção, após a sua incisão em cruz, permite melhor umbilicação e adesão cicatricial. A aplicação dos 6 pontos isolados segundo um hexágono tem dois aspectos: o tipo 1 determina a umbilicação circular quando os pontos são aplicados com precisão nos ângulos do hexágono de lados equidistantes (Figuras 6 e 7); o tipo 2, quando os dois pontos no eixo vertical estão mais afastados, neste último caso, o formato do umbigo fica mais alongado verticalmente (Figuras 8 e 9). Esta dualidade está vinculada ao biótipo das pacientes, sendo aplicado o tipo 1 nas brevilíneas e o tipo 2 nas longilíneas.

Comparando a lipectomia abaixo da posição do umbigo utilizada por Baroudi ${ }^{16}$, Ribeiro ${ }^{17}$, Massiha et al. ${ }^{18}$, LópezTallaj \& Gervain ${ }^{19}$, acreditamos obter um melhor aspecto estético e melhor umbilicação, preservando a gordura divulsionada.

\section{CONCLUSÕES}

A neo-onfaloplastia com preservação da continuidade da pele, de fácil execução técnica, sem cicatriz e com umbilicação natural, tem justificado o seu uso rotineiro nos nossos casos operados de abdominoplastia nos últimos nove anos.
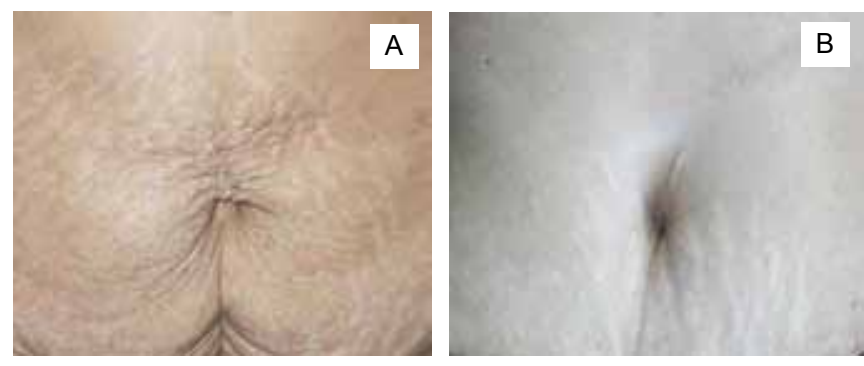

Figura 9 - A e B: Paciente de 35 anos de idade, foto aproximada de pré e $30^{\circ}$ dia de pós-operatório de neoumbilicoplastia tipo 2 , com o eixo vertical mais alongado.

\section{REFERÊNCIAS}

1. Andrews JM. Nova técnica de lipectomia e onfaloplastia. Anais III Congresso Latino-Americano de Cirurgia Plástica;1956. p.148.

2. Avelar JM. Umbilical scar: importance and technique for abdominoplasty. Rev Bras Cir. 1979;2:41.

3. Avelar JM. Abdominoplasty: systematization of a technique without external umbilical scar. Aesthetic Plast J. 1978;2:141-51.

4. Castañares S, Goethel JA. Abdominal lipectomy: a modification in technique. Plast Reconstr Surg. 1967;40(4):378-83.

5. Chicralla A. Onfaloplastia pura e nas abdominoplastias. Rev Méd Guanabara. 1972;39:44.

6. Dubou R, Ousterhout DK. Placement of the umbilicus in an abdominoplasty. Plast Reconstr Surg. 1978;61(2):291-3.

7. Sinder R. Variações técnicas das abdominoplastias. Generalidades sobre cirurgia plástica do abdome. Anais do Simpósio Brasileiro de Abdominoplastias. Rio de Janeiro; 1982. p.33.

8. Kirianoff TG. Making a new umbilicus when none exists. Plast Reconstr Surg. 1978;61(4):603-4.

9. Schoeller T, Wechselberger G, Otto A, Rainer C, Schwabegger A, Lille S, et al. New technique for scarless umbilicul reinsertion in abdominoplasty procedures. Plast Reconstr Surg. 1998;102(5):1720-3.

10. Santanelli F, Mazzocchi M, Renzi C, Cigna E. Reconstruction of a natural-looking umbilicus. Scand J Plast Reconstr Surg Hand Surg. 2002;36(3):183-5

11. Marconi F. Reconstruction of the umbilicus: a simple technique. Plast Reconstr Surg. 1995;95(6):1115-7.

12. Onishi K, Yang YL, Maruyama Y. A new lunch box-type method in umbilical reconstruction. Ann Plast Surg. 1995;35(6):654-6.

13. Franco T, Franco D. Neoomphaloplasty: an old and new technique. Aesthetic Plast Surg. 1999;23(2):151-4.

14. Amud RJM. Neo-onfaloplastia sem cicatriz. Rev Bras Cir Plast. 2008;23(1):37-40.

15. Bozola AR, Psillakis JM. Abdominoplasty: a new concept and classification for treatment. Plast Reconstr Surg. 1988;82(6):983-93.

16. Baroudi R. Umbilicaplasty. Clin Plast Surg. 1975;2(3):431-48.

17. Ribeiro L. Tratamento especifico de gordura nas abdominoplastias. Anais do Simpósio Brasileiro de Abdominoplastias. Rio de Janeiro;1982. p.41-3.

18. Massiha $\mathrm{H}$, Montegut W, Phillips R. A method of reconstructing a naturallooking umbilicus in abdominoplasty. Ann Plast Surg. 1997;38(3):228-31.

19. López-Tallaj L, Gervain J. Restauração umbilical na abdominoplastia: uma simples técnica retangular. Rev Soc Bras Cir Plast. 2001;16(3):39-46. 